\title{
Use of Human Intestinal Enteroids to Detect Human Norovirus Infectivity
}

\author{
Martin Chi-Wai Chan, Sarah K.C. Cheung, \\ Kirran N. Mohammad, Jenny C.M. Chan, \\ Mary K. Estes, Paul K.S. Chan
}

Tools to detect human norovirus infectivity have been lacking. Using human intestinal enteroid cultures inoculated with GII.Pe-GII.4 Sydney-infected fecal samples, we determined that a real-time reverse transcription PCR cycle threshold cutoff of 30 may indicate infectious norovirus. This finding could be used to help guide infection control.

$\mathrm{H}$ uman norovirus accounts for $18 \%$ of acute gastroenteritis cases worldwide (1). Molecular nucleic acid tests, such as real-time reverse transcription PCR (rRTPCR), are widely used for laboratory diagnosis of norovirus RNA in clinical samples (2). These molecular assays are virus specific and their analytical sensitivity is high, but they cannot distinguish between infectious and noninfectious viruses. To study the correlation between viral load measured by rRT-PCR and virus infectivity, we used a recently developed human intestinal enteroid (HIE) culture system (cultures that contain multiple intestinal epithelial cell types) for human norovirus. Ethics approval for this study was obtained from the Joint Chinese University of Hong Kong-New Territories East Cluster Clinical Research Ethics Committee (reference no. 2016.516).

\section{The Study}

We examined the infectivity of human pandemic norovirus genogroup II genotype 4 (GII.Pe-GII.4 Sydney) strains at different inoculating levels by using the adult stem cell-derived HIE line J2 as described previously (3). We seeded enteroid monolayers on 96-well cell culture plates at a density of $6-8 \times 10^{4}$ cells/well and maintained them in differentiation media for 5 days before being inoculated with norovirus. We used fecal samples from 3 norovirus-positive children and from adults in our norovirus surveillance program in Hong Kong (4). We prepared $1 \%$ fecal suspensions and filtered them once by

Author affiliations: The Chinese University of Hong Kong, Hong Kong, China (M.C.-W. Chan, S.K.C. Cheung, K.N. Mohammad, J.C.M. Chan, P.K.S. Chan); Baylor College of Medicine, Houston, Texas, USA (M.K. Estes)

DOI: https://doi.org/10.3201/eid2509.190205 using $0.22-\mu \mathrm{m}$ centrifugal filters, then prepared 3-fold serial dilutions of fecal filtrates in infection medium and stored them at $-70^{\circ} \mathrm{C}$ in multiple aliquots for single use. These dilutions mimicked a broad range of cycle threshold $\left(\mathrm{C}_{\mathrm{t}}\right)$ values of a widely used diagnostic rRT-PCR to represent high to very low norovirus levels (5). We used $33 \mu \mathrm{L}$ of each dilution of fecal filtrate (brought to 100 $\mu \mathrm{L}$ in infection medium) for inoculation of each dilution and performed all virus inoculation steps and downstream cell cultures on enteroids with $\leq 20$ passages in the presence of bile acid (glycochenodeoxycholic acid). We measured norovirus RNA levels in supernatant at 1,24 , and $72 \mathrm{~h}$ after inoculation by using rRT-PCR with a 10 -fold serially diluted standard of in vitro-transcribed norovirus RNA. We considered $a \geq 10$-fold increase in RNA level at $72 \mathrm{~h}$ after inoculation from baseline $(1 \mathrm{~h}$ after inoculation) to indicate productive viral replication and to confirm the presence of infectious virus. We also subjected fecal filtrate dilutions to norovirus antigen detection by use of the Food and Drug Administration-cleared commercial RIDASCREEN Norovirus 3rd Generation EIA (R-Biopharm AG, https://clinical.r-biopharm.com), according to the manufacturer's instructions. We used the same amount of fecal filtrate for inoculation into HIE and EIA measurement and compared the analytical sensitivity of HIE and EIA.

We selected 3 strains of norovirus GII.Pe-GII.4 Sydney, and all replicated productively in HIE line J2; the maximum fold increase of norovirus RNA ranged from 120 $\left(2.1 \log _{10}\right)$ to $45,793\left(4.7 \log _{10}\right)$. Higher levels of replication were obtained from fecal samples from adults (Figure 1). We identified no virus replication when cells were inoculated with fecal filtrate dilutions with $C_{t} \geq 30$. The $C_{t}$ values of the inoculating virus dilution that exhibited a transition from a positive-to-negative enteroid culture result (i.e., having a 10-fold RNA increase) were 27.7, 29.0, and 30.0 for each of the 3 strains. By using linear regression analysis on pooled data of the 3 strains, we estimated that a $\mathrm{C}_{t}$ cutoff of $\leq 30.1$ in inoculating fecal filtrates would indicate the ability to generate productive norovirus GII.Pe-GII.4 Sydney replication (i.e., containing infectious norovirus) (Figure 2).

From 2014 through 2018, a total of 114 (6.5\%) of 1,754 norovirus-positive fecal samples from patients admitted to the Prince of Wales Hospital, Sha Tin, Hong Kong, with 

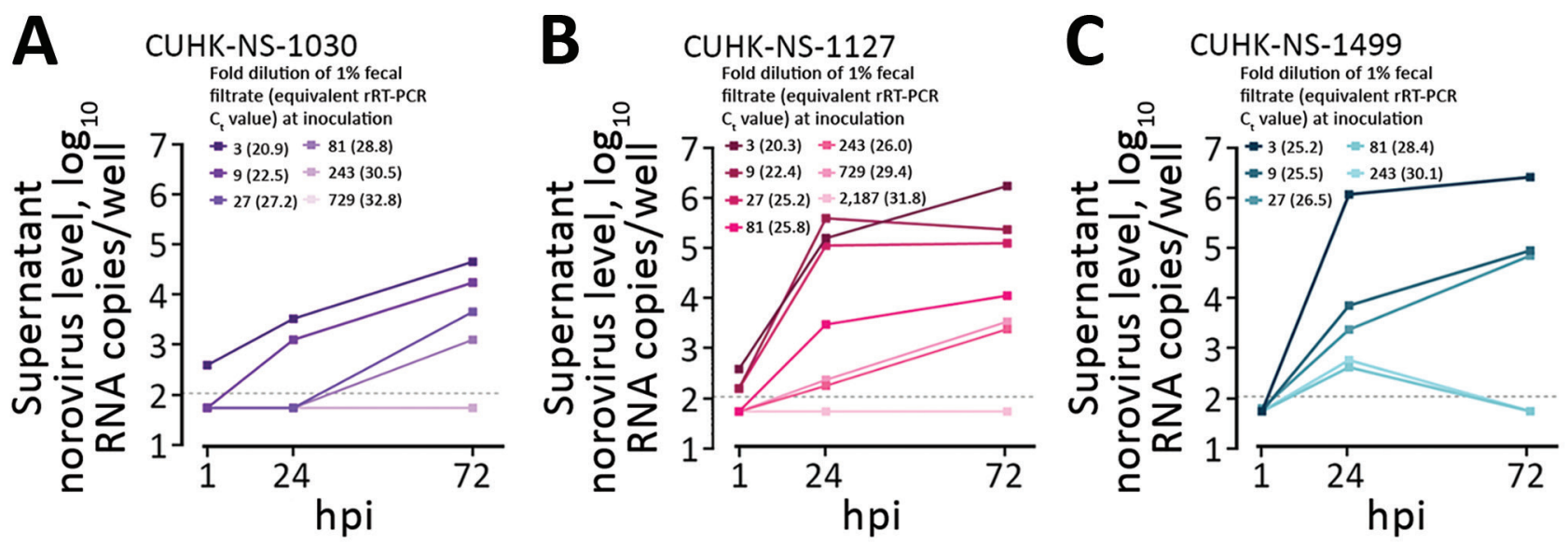

Figure 1. Performance of a human intestinal enteroid (HIE) line and an antigen-based enzyme immunoassay (EIA) to detect human norovirus in clinical fecal samples from 3 patients in Hong Kong. Replication kinetics of 3 human pandemic norovirus genogroup II genotype 4 (GII.Pe-GII.4 Sydney) strains (CUHK-NS-1030, from a 1-year-old boy; CUHK-NS-1127, from a 79-year-old man; CUHKNS-1499, from a 46-year-old man) were tested in a monolayer culture of the adult stem cell-derived HIE line J2. We used 3-fold serial dilutions of norovirus-containing fecal filtrates to challenge J2 seeded on 96-well cell culture plates. Norovirus RNA levels in the culture supernatant at the indicated times were measured by rRT-PCR by using a 10-fold serially diluted standard of in vitro-transcribed norovirus RNA of genotype GII.Pe-GII.4 Sydney. Horizontal dotted lines denote the lower limit of detection of the rRT-PCR (13.8 RNA copies/reaction or 110 RNA copies/well) as determined by probit analysis. Samples with undetectable norovirus RNA were arbitrarily assigned a value equal to half of the lower limit of detection for calculation purpose. To convert the unit from RNA copies/well to RNA copies/mL, multiply the value by a factor of 10 . hpi, hours postinoculation; rRT-PCR, real-time reverse transcription PCR.

acute gastroenteritis had $\mathrm{C}_{\mathrm{t}}$ values $>30\left(\mathrm{C}_{\mathrm{t}}\right.$ median 17.8 ; interquartile range 14.8-22.3; range 5.5-39.2). Among the $1,579(90.0 \%)$ genotyped samples, the proportion of GII.4 was $49.1 \%$; other GII, $44.8 \%$; GI, $5.2 \%$; and co-infections with $>1$ norovirus capsid genotype, $0.9 \%$. Analytical sensitivity of virus replication in HIEs for measuring moderate norovirus shedding was higher than that of EIA by being able to detect infectious virus in fecal filtrate dilutions with $\mathrm{C}_{\mathrm{t}}$ values of 25-30 (Table).

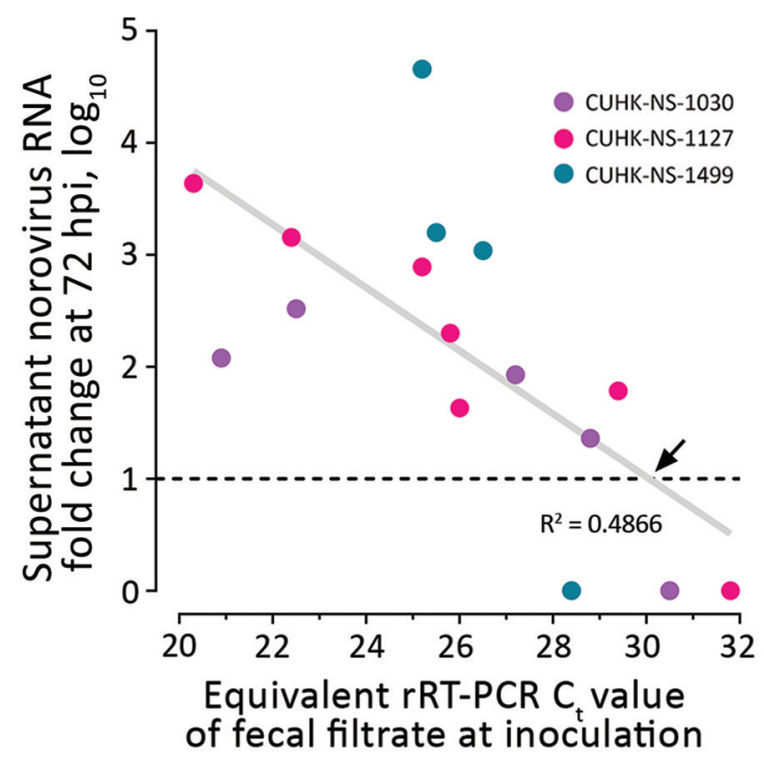

\section{Conclusions}

The limitations of highly sensitive molecular nucleic acid tests and the clinical value of proving virus infectivity were demonstrated in a recent study of Zika virus infection that found that the virus could be detected by culturing samples with high viral RNA levels only (6). For human norovirus, laboratory tools to detect its infectivity have been lacking. Recent technologic advancement in culturing human norovirus in HIE provides a chance to

Figure 2. Estimation of a cutoff for rRT-PCR Ct value of inoculating fecal filtrate indicative of the ability to generate productive norovirus replication (i.e., containing infectious norovirus) in a human intestinal enteroid (HIE) line. We tested 3 strains of pandemic human norovirus genogroup II genotype 4 (GII.Pe-GIl.4 Sydney) (CUHK-NS-1030, from a 1-year-old boy; CUHK-NS-1127, from a 79-year-old man; CUHK-NS-1499, from a 46-year-old man). We used 3-fold serial dilutions of norovirus-containing fecal filtrates to inoculate a monolayer culture of the adult stem cell-derived HIE line J2 seeded on 96-well cell culture plates. Productive norovirus replication was defined as having a supernatant viral RNA level increase of $\geq 10$-fold at $72 \mathrm{hpi}$ from baseline (1 hpi) (horizontal dotted line). RNA fold change data were derived from those shown in Figure 1 and were arbitrarily defined as 1 for those without observable viral RNA replication. For each strain, only the first dilution that resulted in undetectable viral RNA is shown and was included in downstream data analysis. The diagonal gray line denotes the best-fit line from linear regression, and the equation is $Y=-0.2818 X+9.47$, where $Y$ represents RNA fold change and $X$ represents $C_{t}$ value. The black arrow specifies $a_{t}$ cutoff of 30.1 of inoculating fecal filtrate that yields productive norovirus replication as estimated from regression analysis. $C_{t}$, cycle threshold; CUHK, Chinese University of Hong Kong; hpi, hours postinoculation; rRT-PCR, real-time reverse transcription PCR. 
Table. Comparison of rRT-PCR, HIE, and EIA used to detect human norovirus in samples from 3 patients in Hong Kong*

\begin{tabular}{|c|c|c|c|c|}
\hline Isolate† & Fold dilution of $1 \%$ fecal filtrate & rRT-PCR $C_{t}$ & HIE (fold change at $72 \mathrm{hpi}$ ) & $\mathrm{EIA}$ \\
\hline \multirow{6}{*}{ CUHK-NS-1030 } & 3 & 20.9 & $+(120)$ & + \\
\hline & 9 & 22.5 & $+(331)$ & $+/-$ \\
\hline & 27 & 27.2 & $+(85)$ & - \\
\hline & 81 & 28.8 & $+(23)$ & - \\
\hline & 243 & 30.5 & - (ND) & - \\
\hline & 729 & 32.8 & $-(\mathrm{ND})$ & - \\
\hline \multirow[t]{7}{*}{ CUHK-NS-1127 } & 3 & 20.3 & $+(4,362)$ & + \\
\hline & 9 & 22.4 & $+(1,436)$ & + \\
\hline & 27 & 25.2 & $+(781)$ & + \\
\hline & 81 & 25.8 & $+(200)$ & - \\
\hline & 243 & 26.0 & $+(43)$ & - \\
\hline & 729 & 29.4 & $+(61)$ & - \\
\hline & 2,187 & 31.8 & - (ND) & - \\
\hline \multirow[t]{5}{*}{ CUHK-NS-1499 } & 3 & 25.2 & $+(45,793)$ & + \\
\hline & 9 & 25.5 & $+(1,587)$ & + \\
\hline & 27 & 26.5 & $+(1,094)$ & - \\
\hline & 81 & 28.4 & $-(N D)$ & - \\
\hline & 243 & 30.1 & $-(\mathrm{ND})$ & - \\
\hline \multicolumn{5}{|c|}{$\begin{array}{l}{ }^{*} \text { Results for HIE line J2 and the antigen-based RIDASCREEN Norovirus 3rd Generation EIA (R-Biopharm AG, https://clinical.r-biopharm.com) were } \\
\text { compared with the rRT-PCR that had been widely used as a standard for clinical diagnostics of norovirus infections. The same amount of fecal filtrate was } \\
\text { used in HIE line J2 inoculation and EIA measurement at the indicated dilutions. In HIE, numbers indicated fold changes of norovirus RNA level in culture } \\
\text { supernatant at } 72 \text { hpi from baseline (1 hpi); an increase of } \geq 10 \text {-fold was used to confirm the presence of infectious norovirus. Ct, cycle threshold; CUHK, } \\
\text { Chinese University of Hong Kong; EIA, enzyme immunoassay; HIE, human intestinal enteroid; hpi, hours postinoculation; ND, not detectable; rRT-PCR, } \\
\text { real-time reverse transcription PCR; +, positive as defined for each assay; -, negative as defined for each assay; +/-, equivocal as defined according to } \\
\text { the manufacturer's instructions of the EIA. } \\
\text { †CUHK-NS-1030, from a 1-year-old boy; CUHK-NS-1127, from a 79-year-old man; CUHK-NS-1499, from a 46-year-old man. }\end{array}$} \\
\hline
\end{tabular}

examine norovirus infectivity in laboratory settings (3). Using pandemic norovirus GII.Pe-GII.4 Sydney strains, we showed that the most sensitive method for detecting human norovirus was rRT-PCR, followed by HIE infection; EIA was the least sensitive. We experimentally determined that norovirus inocula with $\mathrm{C}_{t} \leq 30$ can robustly yield productive virus replication in HIE, suggesting the presence of infectious virus. This value concurs with findings of a study that proposed an optimal $\mathrm{C}_{\mathrm{t}}$ cutoff of 31 when attributing a disease to norovirus by comparing between symptomatic and asymptomatic cases (7). Our findings imply that a small proportion $(\approx 6.5 \%)$ of patients shedding low levels of norovirus RNA $\left(\mathrm{C}_{\mathrm{t}}>30\right)$ may not be infectious.

In an experimental human volunteer challenge study with norovirus GII.4, low levels of viral RNA shedding were found 10 days after challenge (8). HIE infection can now be used to reevaluate archived samples to better define parameters that would correlate with the infectious period of norovirus gastroenteritis to guide infection control. Of note, in a meta-analysis, the prevalence of norovirus RNA shedding from asymptomatic patients was estimated to be $\approx 9 \%(9)$. Moreover, in a large-scale study of outbreak data from CaliciNet (https://www.cdc. gov/norovirus/reporting/calicinet/index.html), a national norovirus surveillance network in the United States, the median $\mathrm{C}_{\mathrm{t}}$ value among asymptomatic patients was 28 (10). We hypothesize that a substantial proportion of asymptomatic patients are shedding infectious norovirus and that their role in spreading the virus merits our attention. We have shown that high levels of norovirus replication can be achieved from fecal samples of adults, not just young children, in HIE cultures (11).

The use of a fixed $\mathrm{C}_{t}$ cutoff in clinical context needs to be interpreted with caution. First, neither a standardized protocol to perform rRT-PCRs nor a World Health Organization International Standard for norovirus is available to harmonize assay variability across laboratories worldwide. Differences in recovery among nucleic acid extraction methods may further complicate reproducible determination of $\mathrm{C}_{\mathrm{t}}$ values. Second, virus culture systems in cell lines generally lack sensitivity (12), and that of HIE remains unknown. However, it is probably not optimal because input genome equivalents for norovirus to achieve replication are not extremely low $\left(50 \%\right.$ infectious dose $4.4 \times 10^{2}$ to $2.1 \times 10^{3}$ copies/well) $(3,11)$. It is possible that samples with $\mathrm{C}_{\mathrm{t}}>30$ might still contain infectious virus and that low amounts of replicating norovirus would only be detected with further serial propagation of the virus. Third, norovirus replication varies between samples and virus genotypes in HIE culture (11).

In summary, we demonstrate that a $\mathrm{C}_{t}$ cutoff of 30 for a widely used clinical diagnostic rRT-PCR can indicate the presence of infectious GII.Pe-GII.4 Sydney norovirus in an HIE culture model. Patients shedding low levels of norovirus RNA may not be infectious, which should be considered both for estimation of attributable norovirus burden and for clinical management of viral gastroenteritis.

This work was supported in part by the Hong Kong Research Grants Council (to M.C.-W.C.; reference no. 14162217), a seed fund for gut microbiota research by the Faculty of Medicine of 
the Chinese University of Hong Kong (to P.K.S.C.), and a Public Health Service grant (to M.K.E.; reference no. PO1 AI057788).

M.K.E. is named as an inventor on patents related to cloning of the Norwalk virus genome and is a consultant to and has received support from Takeda Vaccines, Inc. Other authors have no conflict of interests to declare.

\section{About the Author}

Dr. M.C.-W. Chan is an assistant professor in the Department of Microbiology and Stanley Ho Centre for Emerging Infectious Diseases and a principal investigator in the Li Ka Shing Institute of Health Sciences of the Chinese University of Hong Kong. His research interest focuses on molecular epidemiology and pathogenesis of human noroviruses; other research interests include use of metagenomic next-generation sequencing in clinical diagnostics, gut virome, and food virology.

\section{References}

1. Ahmed SM, Hall AJ, Robinson AE, Verhoef L, Premkumar P, Parashar UD, et al. Global prevalence of norovirus in cases of gastroenteritis: a systematic review and meta-analysis. Lancet Infect Dis. 2014;14:725-30. https://doi.org/10.1016/ S1473-3099(14)70767-4

2. Vinjé J. Advances in laboratory methods for detection and typing of norovirus. J Clin Microbiol. 2015;53:373-81. https://doi.org/10.1128/JCM.01535-14

3. Ettayebi K, Crawford SE, Murakami K, Broughman JR, Karandikar U, Tenge VR, et al. Replication of human noroviruses in stem cell-derived human enteroids. Science. 2016;353:1387-93. https://doi.org/10.1126/science.aaf5211

4. Chan MC, Kwok K, Zhang LY, Mohammad KN, Lee N, Lui GCY, et al. Bimodal seasonality and alternating predominance of norovirus
GII.4 and non-GII.4, Hong Kong, China, 2014-2017. Emerg Infect Dis. 2018;24:767-9. https://doi.org/10.3201/eid2404.171791

5. Kageyama T, Kojima S, Shinohara M, Uchida K, Fukushi S, Hoshino FB, et al. Broadly reactive and highly sensitive assay for Norwalk-like viruses based on real-time quantitative reverse transcription-PCR. J Clin Microbiol. 2003;41:1548-57. https://doi.org/10.1128/JCM.41.4.1548-1557.2003

6. Mead PS, Duggal NK, Hook SA, Delorey M, Fischer M, Olzenak McGuire D, et al. Zika virus shedding in semen of symptomatic infected men. N Engl J Med. 2018;378:1377-85. https://doi.org/10.1056/NEJMoa1711038

7. Phillips G, Lopman B, Tam CC, Iturriza-Gomara M, Brown D, Gray J. Diagnosing norovirus-associated infectious intestinal disease using viral load. BMC Infect Dis. 2009;9:63. https://doi.org/10.1186/1471-2334-9-63

8. Bernstein DI, Atmar RL, Lyon GM, Treanor JJ, Chen WH, Jiang $X$, et al. Norovirus vaccine against experimental human GII.4 virus illness: a challenge study in healthy adults. J Infect Dis. 2015;211:870-8. https://doi.org/10.1093/infdis/jiu497

9. Qi R, Huang YT, Liu JW, Sun Y, Sun XF, Han HJ, et al. Global prevalence of asymptomatic norovirus infection: a meta-analysis. EClinicalMedicine. 2018;2-3:50-8. https://doi.org/10.1016/ j.eclinm.2018.09.001

10. Shioda K, Barclay L, Becker-Dreps S, Bucardo-Rivera F, Cooper PJ, Payne DC, et al. Can use of viral load improve norovirus clinical diagnosis and disease attribution? Open Forum Infect Dis. 2017;4:ofx131. https://doi.org/10.1093/ofid/ofx131

11. Costantini V, Morantz EK, Browne H, Ettayebi K, Zeng XL, Atmar RL, et al. Human norovirus replication in human intestinal enteroids as model to evaluate virus inactivation. Emerg Infect Dis. 2018;24:1453-64. https://doi.org/10.3201/eid2408.180126

12. Leland DS, Ginocchio CC. Role of cell culture for virus detection in the age of technology. Clin Microbiol Rev. 2007;20:49-78. https://doi.org/10.1128/CMR.00002-06

Address for correspondence: Martin C.-W. Chan, Prince of Wales Hospital, Department of Microbiology, 1/F, Lui Che Woo Clinical Sciences Bldg, Shatin, Hong Kong, China; email: martin.chan@cuhk.edu.hk

\title{
etymologia
}

Etymology is concerned with the origin of words, how they ve evolved over time, and changed in form and meaning as they were translated from one language to another. Every month, EID publishes a feature highlighting the etymology of a word from medicine or public health.

\section{featured monthly in EMERCING
INFECTIOUS DISEASES}

\author{
http://wwwnc.edc.gov/eid/articles/etymologia
}

